\title{
Jean-Paul Marthoz, Journalisme international
}

Bruxelles, Éd. De Boeck Université, 2008

\section{Mélanie Gérard}

\section{OpenEdition}

\section{Journals}

Édition électronique

URL : http://journals.openedition.org/questionsdecommunication/950

DOI : 10.4000/questionsdecommunication.950

ISSN : 2259-8901

\section{Éditeur}

Presses universitaires de Lorraine

\section{Édition imprimée}

Date de publication : 1 juillet 2009

Pagination : 451-452

ISBN : 978-2-86480-989-0

ISSN : $1633-5961$

\section{Référence électronique}

Mélanie Gérard, « Jean-Paul Marthoz, Journalisme international », Questions de communication [En ligne], 15 | 2009, mis en ligne le 17 janvier 2012, consulté le 22 septembre 2020. URL : http:// journals.openedition.org/questionsdecommunication/950 ; DOI : https://doi.org/10.4000/ questionsdecommunication.950

Ce document a été généré automatiquement le 22 septembre 2020.

Tous droits réservés 


\title{
Jean-Paul Marthoz, Journalisme international
}

\author{
Bruxelles, Éd. De Boeck Université, 2008
}

Mélanie Gérard

\section{RÉFÉRENCE}

Jean-Paul Marthoz, Journalisme international. Bruxelles, Éd. De Boeck Université, coll. Info Com, 2008, $279 \mathrm{p}$.

1 Journaliste et enseignant, Jean-Paul Marthoz s'est fondé sur sa propre expérience et celles d'une dizaine de reporters pour livrer aux étudiants et professeurs en journalisme sa conception du Journalisme international qui complète celle développée dans Et maintenant le monde en bref. Les médias et le nouveau désordre mondial (Bruxelles, Éd. Complexe, 2006). L'exposé, qui retrace les évolutions du journalisme international dans un contexte politique, culturel et économique, se veut résolument pédagogique. Le lecteur y trouvera une abondance de ressources et outils très concrets pour informer sur le monde à l'étranger. Par ailleurs, la démarche engagée de l'auteur, pour qui le journalisme est aussi une mission, invite l'étudiant à une réflexivité sur sa propre conception du métier en l'incitant à se définir en tant que journaliste.

2 Selon Jean-Paul Marthoz, le journalisme international doit relever de lourds défis : la globalisation entraîne des imbrications de plus en plus étendues entre les sociétés à tous les niveaux et, paradoxalement, ce mouvement s'accompagne d'une fragmentation du monde en différentes groupes qui se replient sur leurs identités. Les populations en ont fait le douloureux constat avec les attentats de New York, de Londres ou de Madrid. Les décisions prises par la Commission européenne ont des répercussions directes sur le quotidien des individus, les maladresses de quelques financiers américains affectent l'économie mondiale... Or, malgré ces nouvelles proximités, l'actualité internationale ne suscite qu'une attention relative de la part du public qui continue à préférer l'information locale. Dans les médias, le corollaire de ce désintérêt se caractérise par la 
réduction des coûts consacrés au traitement des événements étrangers (réduction de l'espace dans les supports, fermeture des bureaux à l'étranger...).

3 Pour l'auteur, une évidence s'impose : il est nécessaire de repenser le journalisme international dont les pratiques sont aujourd'hui trop désuètes pour rendre compte de la complexité du monde. Il faut « tisser des liens entre le local et le global, l'universel et le particulier, afin de donner du sens à l'actualité » (p. 10), pratiquer un "journalisme d'intérêt public»(p.9) pour y impliquer le citoyen en chaque lecteur. Concernant l'organisation du travail dans les rédactions, il importe de ne plus cloisonner l'actualité internationale au "service étranger » car elle peut concerner tous les aspects de la société (économie, sport, culture...), et de favoriser la coopération entre les différents services. Les dirigeants des médias doivent faire preuve d'audace en imitant les initiatives prises par les collègues, à l'instar de la revue Enjeux internationaux qui a adopté la "glocalisation » (p. 121) : traiter l'information locale dans un contexte global (une rubrique «Proximités » avec le sous-titre "Ça c'est passé loin de chez vous et ça vous concerne»). Il faut étendre le journalisme transnational qui implique la participation de plusieurs correspondants étrangers travaillant pour différents médias afin de faciliter le traitement des sujets qui dépassent les frontières comme l'immigration, les trafics de stupéfiants ou le blanchiment d'argent sale. Et si l'auteur est convaincu de la nécessaire réforme des pratiques du journalisme international, il est également convaincu par sa faisabilité, et ce, malgré les contraintes économiques qui pèsent sur les médias. L'influence des publicitaires, le processus de concentration et de conglomération (l'entrée de groupes économiques et financiers dans le secteur) affectent certes la gestion commerciale des entreprises de presse et leur choix rédactionnels, mais ils ne sont pas un obstacle infranchissable.

On l'aura compris, l'ambition de Jean-Paul Marthoz va au-delà d'une refonte des pratiques, il s'agit de revoir les fondements du journalisme international, dont « l'enjeu [serait] d'apporter une valeur ajoutée à l'information qui tend à s'homogénéiser, de trouver des angles qui permettent aux médias non seulement d'expliquer la complexité du monde, mais aussi de marquer leur différence par rapport à leurs concurrents » (p.112). Pourtant, ces médias existent déjà de par le monde et en lisant leur recensement (non exhaustif) établi par l'auteur, on serait presque surpris par leur nombre. Ce serait oublier qu'il s'agit rarement de médias grand public. L'analyse de la presse américaine qui, selon lui, exerce une forte influence sur l'actualité internationale, pourrait s'appliquer à bien des sociétés: une dualisation du public marquée par une minorité de personnes bien informées et une majorité peu ou mal informées, dualisation qui se reflète dans la presse internationale avec quelques titres prestigieux et une abondance de médias délaissant les événements étrangers.

5 Enfin, dernier défi pour le journaliste, qui est très significatif de la démarche de l'auteur: s'engager à informer, en plus de son devoir de connaissance - tout journaliste compétent se doit de posséder une vaste culture générale -, en s'armant de courage: "Non seulement le courage physique d'affronter les snipers et les tueurs, mais aussi celui de nager à contre- courant des règles de l'information business. Le courage face à sa direction, en se battant pour des sujets qu'aucun responsable de marketing ne s'aviserait de cautionner; le courage face à une opinion publique qui recherche souvent dans les informations la confirmation de ses préjugés et la justification de son indifférence; le courage face à ses pairs, trop souvent tentés par le journalisme grégaire » (p. 183). En faisant ainsi référence à des faits tragiques, tels que 
les conflits armés, les génocides, les guerres ethniques ou les catastrophes humanitaires, l'auteur se positionne par rapport à des questions éthiques et déontologiques. En outre, il considère que, dans ce contexte, les médias ont la responsabilité d'informer le public, de faire fi de leur position de neutralité pour aider les gens à comprendre les enjeux de la situation, et de reconsidérer les critères d'objectivité journalistique si la conscience morale du reporter lui impose de le faire.

6 Avec un investissement plus marqué dans les pays anglo-saxons qu'en France, l'étude du journalisme international dans le domaine des sciences de l'information de la communication s'est peu développée jusqu'à présent. Aussi l'ouvrage de Jean-Paul Marthoz, même s'il n'a pas de visée scientifique à proprement parler, renseigne-t-il de façon détaillée sur certains aspects de l'organisation du travail du reporter se rendant ou séjournant à l'étranger pour couvrir un événement. Ses analyses et ses observations sur les spécialités journalistiques et les acteurs sociaux qui participent à la construction de l'information internationale sont du plus grand intérêt pour tout chercheur se penchant sur cette catégorie professionnelle. Par ailleurs, les propositions formulées par l'auteur concernant la réforme du journalisme international nous paraissent bien ambitieuses au regard des réalités et des possibilités offertes au journaliste pour évoluer vers son "idéal ». Et certaines analyses, à peine amorcées, à propos de l'engagement du journaliste ou encore du traitement journalistique de la personne souffrante, mériteraient d'être développées, surtout pour les lecteurs non initiés auxquelles elles s'adressent. Un défi pour l'auteur?

\section{AUTEURS}

\section{MÉLANIE GÉRARD}

CREM, université Paul Verlaine-Metz

melanigerard@hotmail.fr 